\title{
Pesca con anzuelos en barcos palangreros del Océano Pacífico mexicano: efectos en la captura y peso de tiburones y otras especies
}

\author{
Fishing by hooks in longliners from the Mexican Pacific Ocean: effects in \\ the catch rate and weight of sharks and other species
}

\section{Ildefonso Galeana-Villaseñor ${ }^{1}$, Felipe Galván-Magaña² ${ }^{2}$ Heriberto Santana-Hernández ${ }^{3}$}

\author{
${ }^{1}$ Instituto Tecnológico de Mazatlán. Corsario 1 No.203. Col. Urías. Mazatlán, Sinaloa México. C.P. 82070 \\ ${ }^{2}$ Centro Interdisciplinario de Ciencias Marinas. Av. IPN s/n. Apdo. Postal 592. La Paz, México. C.P. 23000 \\ ${ }^{3}$ Centro Regional de Investigación Pesquera. Playa Ventana S/N. Apdo. Postal 591 Manzanillo, Col. México. C.P. 28200 \\ galvan.felipe@gmail.com
}

\begin{abstract}
We analyzed the effects of two hook types and two bait types on the catch rate of long liners from the northwest Pacific Ocean off Mexico. In 47 sets, from four commercial fishing trips done during March 2005 to August 2006, we used conventional J-style $9 / 011^{\circ}$ offset hooks and experimental circle 16/0 $0^{\circ}$ offset hooks. We used two bait types: jumbo squid (Dosidicus gigas) and striped mullet juveniles (Mugil cephalus). A factorial experiment split-plot design was applied. A total of 1304 organisms were caught, where $86.5 \%$ were sharks, and blue shark (Prionace glauca) was the dominant specie and $13.5 \%$ from other species. While the hook type did not affect significantly the catch rate and weight of sharks and other species, the bait type had a significant effect, mainly on the catch rate of blue shark, silky shark (Carcharhinus falciformis), dolphinfish (Coryphaena hippurus), and the weight of total catch. The Tukey test showed that the mean catch rate of blue shark, silky shark, dolphinfish, total catch and weight were higher for squid bait than for striped mullet.
\end{abstract}

Key words: By-catch, blue shark, Eastern Pacific Ocean
Resumen.- Se evaluó el efecto de dos tipos de anzuelos y dos tipos de carnada en la tasa de captura de barcos palangreros en el noroeste del Océano Pacífico mexicano. Se realizaron cuatro viajes de pesca comercial con un total de 47 lances, entre marzo de 2005 y agosto de 2006. Los dos tipos de anzuelos usados fueron: recto $11^{\circ}$ de inclinado $9 / 0$ de uso tradicional y circular plano 16/0 (experimental). Se utilizaron dos tipos de carnada: calamar gigante (Dosidicus gigas) y peces juveniles de lisa (Mugil cephalus). Se aplicó un diseño experimental factorial en parcelas divididas ('split-split-plot'). Se capturó un total de 1304 ejemplares, de los cuales 86,5\% fueron tiburones, siendo el tiburón azul (Prionace glauca) la especie dominante y $13,5 \%$ estuvo constituido por otras especies. Los resultados mostraron que el tipo de anzuelo no presentó diferencias significativas en la tasa y peso de la captura de tiburones y de las otras especies consideradas como pesca incidental; el tipo de carnada presentó un efecto significativo en la tasa de captura, principalmente en tiburón azul, tiburón jaquetón (Carcharhinus falciformis), dorado (Coryphaena hippurus), así como en el peso de la captura total. La prueba de Tukey, mostró que la carnada de calamar presentó una media superior a la carnada de lisa en la tasa de captura del tiburón azul, piloto, dorado, captura y peso total.

Palabras clave: Pesca incidental, tiburón azul, Océano Pacífico oriental

unidades de pesquerías: la ribereña artesanal que se realiza a lo largo de los litorales marinos con embarcaciones menores de 10,5 m de eslora, contribuyendo con el $40 \%$ de la producción nacional; la de mediana altura, que se realiza con embarcaciones de 10 a $27 \mathrm{~m}$ de eslora en aguas costeras, y la pesca de altura, en donde se utilizan embarcaciones de más de $27 \mathrm{~m}$ de eslora, tanto en aguas costeras como aguas oceánicas dentro de la zona económica exclusiva del océano Pacífico. Los últimos dos tipos de pesca aportan el restante 60\% de la producción nacional (CONAPESCA-INP 2004, Diario Oficial de la Federación 2007). 
En los últimos años se han adaptado barcos de la flota de arrastre camaronera del Pacífico mexicano, con tambores de accionamiento hidráulico y dispositivos para operar palangres pelágicos de hasta $55 \mathrm{~km}$ de longitud, los cuales se incorporan a la pesca de tiburón, de enero a julio, cuando ha disminuido la captura de camarón o se encuentra en veda; esto ha incrementado el esfuerzo de la pesca dirigida a tiburones y a la captura de otras especies como el marlín azul (Makaira nigricans), marlín rayado (Tetrapturus audax), pez vela (Istiophorus platypterus) y dorado que, de acuerdo con la Ley de Pesca de México, están reservadas para la pesca deportiva (Secretaría de Medio Ambiente Recursos Naturales y Pesca 1999). La flota comercial palangrera mexicana dirigida a la captura de pelágicos mayores comparte este recurso con la pesca deportiva, lo que ha generado conflictos de intereses entre estos dos tipos de pesquería, manifestándose en inconformidades y presión de tipo político, económico y social hacia los encargados de la administración de las pesquerías (Santana-Hernández 2001). Hay un creciente interés en buscar alternativas de solución a este problema dirigidas a maximizar la eficiencia del palangre pelágico para la captura de la especie-objeto, disminuir la pesca incidental y facilitar la regulación del esfuerzo pesquero, por lo que existe interés en realizar estudios que evalúen el efecto que los diferentes componentes del arte de pesca, como el tipo de carnada y el tipo de anzuelo, pueden ejercer en los resultados de la captura.

La efectividad y selectividad del palangre depende de la interrelación de factores tanto ambientales, biológicos, como tecnológicos (Løkkeborg \& Bjordal 1992). Dentro de algunos de estos factores se pueden considerar además el índice lunar y la velocidad del viento que pueden afectar la vulnerabilidad del pez a las operaciones de pesca (Bigelow et al. 1999), la profundidad de captura del palangre en relación a la distribución vertical de las especies objeto de captura y las incidentales (Boggs 1992, Santana-Hernández 1998), los tiempos de operación del calado, cobrado y deriva del palangre (Lokkeborg \& Pina 1997), el tipo de carnada y su posición de encarnado (Broadhusrst \& Hazin 2001), la atracción y comportamiento del pez hacia la carnada (Johnstone \& Hawkins 1981, Kaimmer 1999, Fréon \& Misund 1999), el tipo y tamaño del anzuelo (Kerstetter \& Graves 2006, Yokota et al. 2006).

En la pesquería de palangre pelágico del Océano Pacifico mexicano los pescadores utilizan como carnada calamar (Dosidicus gigas), macarela (Scomber japonicus), lisa (Mugil cephalus) y barrilete (Katsuwonus pelamis). Tradicionalmente utilizan los anzuelos recto, el tipo atunero japonés plano e inclinado, pero por disposición de la norma NOM-029-PESC-2006 de México, se deben usar anzuelos circulares cuando menos en los reinales más cercanos a cada orinque (Diario Oficial de la Federación 2007) considerando que esta medida reduce la pesca incidental. Sin embargo, los pescadores presentan una resistencia natural al uso de este anzuelo por ser un elemento nuevo y extraño a su costumbre de pescar, teniendo la desconfianza de que pueda afectar el rendimiento del palangre en la captura de tiburón. Algo similar está sucediendo en un gran porcentaje en las pesquerías pelágicas internacionales de palangre en el Océano Atlántico, donde se continúa el uso de el anzuelo recto tradicional (Kerstetter \& Graves 2006). Existe una tendencia internacional de uso del anzuelo circular, sin embargo se requiere examinar con mayor amplitud el efecto del anzuelo circular en la eficiencia del palangre sobre la especie objeto de captura (ejemplo: atún, pez espada y tiburón) y cómo las modificaciones del arte de pesca afecta la captura por unidad de esfuerzo (CPUE) de las especies objeto de captura y la pesca incidental (Yokota et al. 2006).

En este estudio se evaluó el efecto del anzuelo recto inclinado tradicional 9/0 en comparación con el anzuelo experimental circular plano 16/0 y dos tipo de carnada, el calamar y la lisa, en la tasa de captura y peso de tiburón y otras especies que se consideran como pesca incidental.

\section{Material y métodos}

El experimento se realizó a bordo de tres barcos camaroneros adaptados con tambores hidráulicos para operar el palangre pelágico en el noroeste del océano Pacífico mexicano (Fig. 1), en cuatro viajes de pesca comercial del 8 de marzo de 2005 al 3 de agosto de 2006. Se realizaron 47 lances de palangre pelágico. El primer viaje correspondió a la temporada invierno-primavera/ 2005, con 5 lances, el segundo a la primavera-verano/ 2005, con 12 lances, el tercero a invierno-primavera/2006, con 24 lances, y el cuarto al verano/2006, con 6 lances. El palangre consistió en una línea madre de poliamida monofilamento de $4 \mathrm{~mm}$ de diámetro y una longitud de $24 \mathrm{~km}$, a la cual se le unieron los reinales aproximadamente cada $50 \mathrm{~m}$ con 5 anzuelos por canasta (Fig. 2A). Los orinques se construyeron con 10 a 15 m de poliamida monofilamento de $3 \mathrm{~mm}$ de diámetro con broches en los extremos para unirlos al flotador y a la línea madre. Los reinales se armaron con dos secciones, la primera donde se une el anzuelo con $50 \mathrm{~cm}$ de alambrada de acero inoxidable con diámetro de 1,6 mm y la segunda con $15 \mathrm{~m}$ de línea de poliamida monofilamento de $3 \mathrm{~mm}$ de diámetro con un broche en el extremo para unirla a la línea madre (Fig. 2B). Se utilizaron dos tipos de anzuelo, uno experimental de forma 


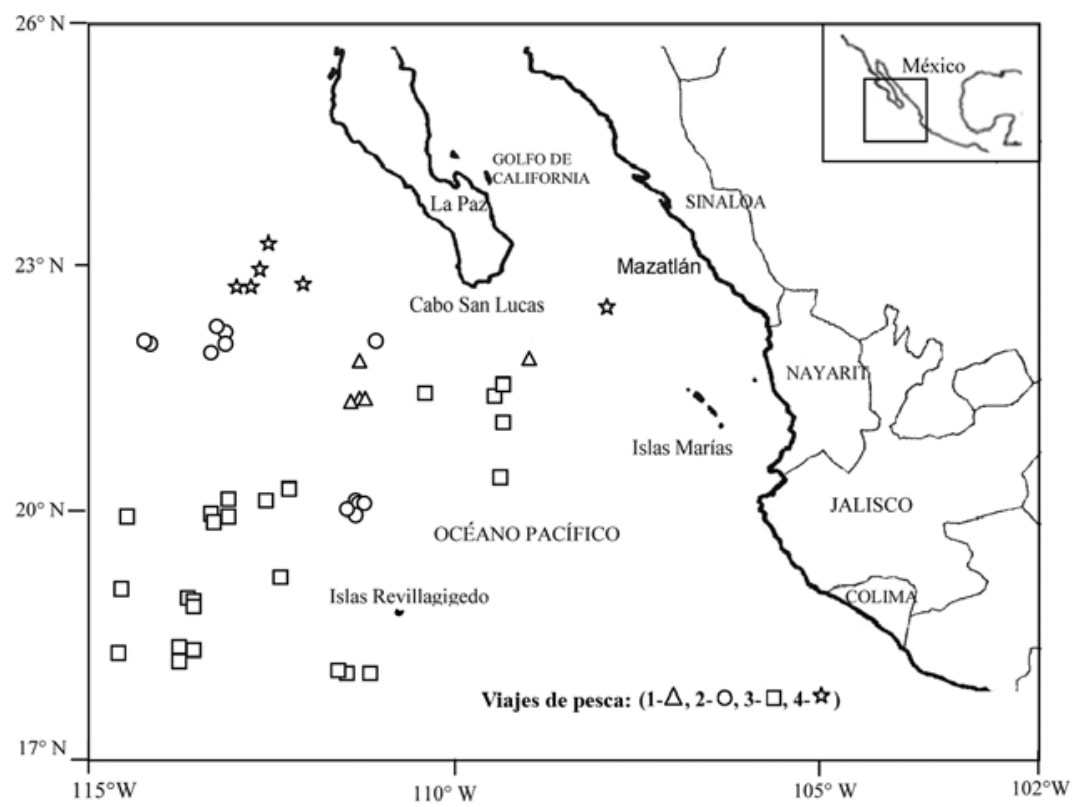

Figura 1

Área de estudio y ubicación de 47 lances del palangre pelágico experimental realizados desde el 8 de marzo, 2005 al 3 de agosto, 2006 en cuatro viajes de pesca en el Océano Pacífico mexicano

Study area showing 47 sets position done from March 8, 2005 to August 3, 2006 in four fishing trips in the Mexican Pacific Ocean

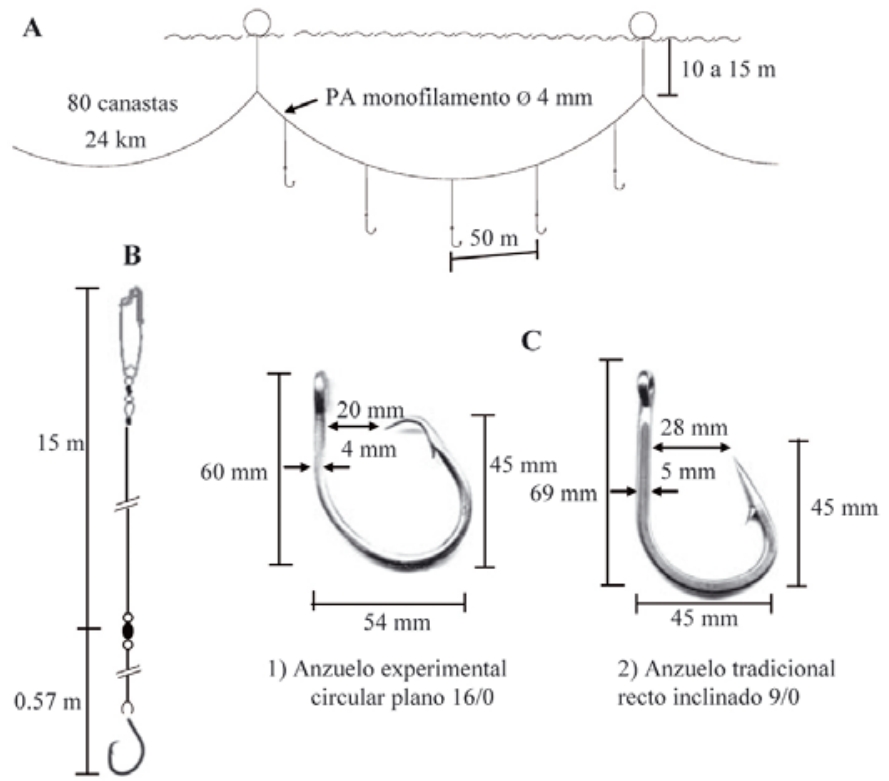

Figura 2

Representación esquemática del palangre pelágico. (A) Sección del palangre, (B) reinal, (C) tipos de anzuelos utilizados en este estudio (1-circular, 2-tradicional)

Schematic representation of a pelagic longline. (A) Longline section, (B) gangions, (C) hook types utilized in this study (1-circular, 2-traditional) 
circular plano 16/0 y otro de forma recta con una inclinación izquierda de $11^{\circ}$ (visto el anzuelo de frente) y tamaño nominal 9/0 que tradicionalmente utilizan los pescadores en el palangre pelágico (Fig. 2C). La carnada que se utilizó fue calamar gigante (Dosidicus gigas) con un peso medio de $400 \mathrm{~g}$ y juveniles de lisa macho (Mugil cephalus) con un peso medio de 180 g. Del calamar se utilizaron secciones de manto y cabeza con tentáculos (Fig. 3). En cada lance se utilizaron 400 carnadas divididas en cuatro canastos de 100 carnadas de un mismo tipo cada uno. Se combinaron 400 anzuelos y 400 carnadas para formar cuatro combinaciones: circular-calamar, circular-lisa, recto-calamar y recto-lisa en paquetes de 100 anzuelos y 100 carnadas cada combinación, los cuatro paquetes se distribuyeron al azar en cada lance.

En cada viaje los lances fueron ubicados geográficamente por medio de un Sistema de Posicionamiento Global (GPS, por sus siglas en inglés) y durante el cobrado del palangre se identificaron las especies capturadas registrándose el número de ejemplares y su peso en kilogramos.

Se utilizó un diseño experimental de parcelas subdivididas ('split-split-plot design') con el siguiente modelo estadístico (Montgomery 1976, Herrera \& Barreras 2001): donde $\mathrm{y}_{\mathrm{ijkh}}$ es la variable respuesta que se consideró a la tasa de captura (número de peces/100 anzuelos) y el peso (kg), ì es la media general, $\mathrm{V}_{\mathrm{i}}, \mathrm{L}_{\mathrm{i}} \mathrm{y}\left(\mathrm{V}^{*} \mathrm{~A}\right)_{\mathrm{ij}}$ representan la parcela completa y corresponden a los viajes (bloques), lance y el error de la parcela completa (interacción viaje*lance) respectivamente; mientras que $A_{k},\left(V^{*} A\right)_{i k}$, $\left(\mathrm{L}^{*} \mathrm{~A}\right)_{\mathrm{jk}} \mathrm{y}\left(\mathrm{V}^{*} \mathrm{~L}^{*} \mathrm{~A}\right)_{\mathrm{ijk}}$ representan la subparcela $\mathrm{y}$ corresponde al anzuelo, interacciones viaje*anzuelo, lance*anzuelo y al error de la subparcela (interacción viaje*lance*anzuelo) respectivamente; $\mathrm{C}_{\mathrm{h}}$ (carnada) y los restantes parámetros corresponden a la sub-subparcela y representan respectivamente la sub-subparcela del tratamiento de la carnada y sus interacciones. La cuarta interacción $\left(\mathrm{V}^{*} \mathrm{~L} * \mathrm{~A} * \mathrm{C}\right)_{\mathrm{ijkh}}$ representa el error para la subsubparcela. De este modelo se consideran todas las variables que puedan tener influencia en la tasa de captura (i.e. viaje (que implícitamente toma la estacionalidad), lance, anzuelo y carnada).

Se utilizó el modelo lineal general (GLM) para el análisis de los datos de la tasa de captura y peso de los organismos capturados. Como la tasa de captura no guardó una distribución normal, se transformó con $\ln (\mathrm{x}+1)$ para inducir normalidad. La comparación de medias se realizó con la prueba de Tukey (Herrera \& Barreras 2001).

$$
\mathrm{y}_{\mathrm{ijkh}}=\grave{\mathrm{i}}+\mathrm{V}_{\mathrm{i}}+\mathrm{L}_{\mathrm{j}}+\left(\mathrm{V}^{*} \mathrm{~L}\right)_{\mathrm{ij}}+\mathrm{A}_{\mathrm{k}}+\left(\mathrm{V}^{*} \mathrm{~A}\right)_{\mathrm{ik}}+\left(\mathrm{L}^{*} \mathrm{~A}\right)_{\mathrm{jk}}+\left(\mathrm{V}^{*} \mathrm{~L}^{*} \mathrm{~A}\right)_{\mathrm{ijk}}+\mathrm{C}_{\mathrm{h}}+\left(\mathrm{V}^{*} \mathrm{C}\right)_{\mathrm{ih}}+\left(\mathrm{L}^{*} \mathrm{C}\right)_{\mathrm{jh}}+\left(\mathrm{V}^{*} \mathrm{~L}^{*} \mathrm{C}\right)_{\mathrm{ijh}}+(\mathrm{A} * \mathrm{C})_{\mathrm{kh}}+\left(\mathrm{V}^{*} \mathrm{~A}^{*} \mathrm{C}\right)_{\mathrm{ikh}}+\left(\mathrm{L}^{*} \mathrm{~A}^{*} \mathrm{C}\right)_{\mathrm{jkh}}+\left(\mathrm{V}^{*} \mathrm{~L}^{*} \mathrm{~A}^{*} \mathrm{C}\right)_{\mathrm{ijkh}}
$$

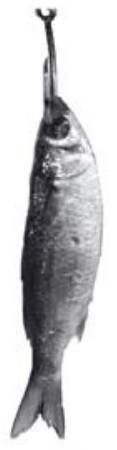

Juvenil de lisa macho (Mugil cephalus Linnaeus, 1758)

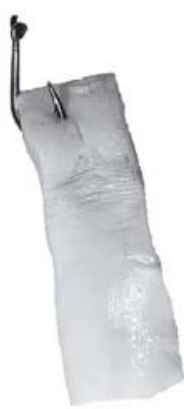

a) Manto

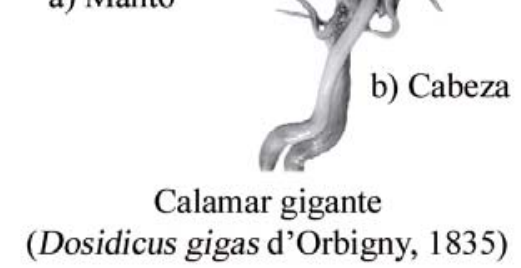

Figura 3

Tipo de carnadas utilizada en el palangre de deriva

Bait type used in the experimental longline 


\section{Resultados}

\section{Composición, esfuerzo y tasa de la captura total}

Se capturó un peso total de $43.719 \mathrm{~kg}$ con 1.304 organismos, los cuales se clasificaron en dos grupos (Tabla 1): El de tiburones que representó el 86,5\% de la captura total con 960 organismos, de los cuales el tiburón azul (Prionace glauca) fue la especie dominante con $85,1 \%$ de su grupo y $73,6 \%$ de la captura total y el grupo de otras especies que representaron el $13,5 \%$ de la captura total, siendo el dorado (Coryphaena hippurus) la especie dominante con $26,7 \%$ de este grupo y $3,6 \%$ de la captura total.

La tasa de captura total presentó su valor máximo (14 organismos/100 anzuelos) en la temporada inviernoprimavera/2005, donde se aplicó el menor valor del esfuerzo (2000 anzuelos), presentando una tendencia de disminución para las siguientes temporadas. El aumento del esfuerzo en la temporada invierno-primavera/06 no se reflejó en un aumento de la tasa de captura, manteniéndose casi estable entre la temporada primaveraverano/05 y verano/06, donde se presentaron variaciones del esfuerzo disminuyendo en la temporada verano/06. Durante 2005 la captura total fue menor $(17,1 \%)$ que en 2006, pero la tasa de captura fue superior en el año 2005 $(45,5 \%)$ con un esfuerzo menor que durante 2006 (76,5\%), mostrando una aparente disminución de la disponibilidad del recurso del 2005 al 2006 con una ligera recuperación para la temporada de invierno-primavera del 2006 (Fig. 4).

Se realizó el análisis solo en los datos de tiburón azul (Prionace glauca), tiburón jaquetón (Carcharhinus falciformis), tiburón mako (Isurus oxyrinchus), dorado (Coryphaena hippurus), marlín rayado (Tetrapturus audax) y marlín azul (Makaira nigricans) por el escaso número de ejemplares capturados de las demás especies.

Tabla 1

Composición del total de especies capturadas con el palangre de deriva

Total catch composition of the pelagic longline

\begin{tabular}{|c|c|c|c|c|}
\hline Grupo & Especie & & $\mathrm{N}^{\circ}$ & Peso $(\mathrm{kg})$ \\
\hline \multirow[t]{12}{*}{ Tiburones } & Tiburón azul & Prionace glauca & 960 & 31.951 \\
\hline & Tiburón jaquetón & Carcharhinus falciformis & 59 & 2.816 \\
\hline & Tiburón mako & Isurus oxyrinchus & 44 & 1.569 \\
\hline & Tiburón zorro pelágico & Alopias pelagicus & 29 & 1.230 \\
\hline & Tiburón grillo & Alopias superciliosus & 13 & 354 \\
\hline & Cornuda prieta & Sphyrna zygaena & 12 & 608 \\
\hline & Tiburón aleta blanca & Carcharhinus longimanus & 3 & 102 \\
\hline & Cornuda gigante & Sphyrna mokarran & 3 & 263 \\
\hline & Cornuda común & Sphyrna lewini & 2 & 103 \\
\hline & Tiburón prieto & Carcharhinus obscurus & 2 & 93 \\
\hline & Tiburón galápago & Carcharhinus galapagensis & 1 & 81 \\
\hline & & Subtotal & 1.128 & 39.170 \\
\hline \multirow[t]{13}{*}{ Otras especies } & Dorado & Coryphaena hippurus & 47 & 472 \\
\hline & Marlín rayado & Tetrapturus audax & 38 & 1.421 \\
\hline & Marlín azul & Makaira nigricans & 36 & 1.450 \\
\hline & Pez espada & Xiphias gladius & 31 & 738 \\
\hline & Guajo & Acanthocybium solandri & 9 & 114 \\
\hline & Atún aleta amarilla & Thunnus albacares & 5 & 117 \\
\hline & Tortuga perico & Careta c. gigas & 4 & 80 \\
\hline & Raya látigo violeta & Dasyatis violacea & 3 & 7 \\
\hline & Manta de aguijón & Mobula japanica & 1 & 80 \\
\hline & Tortuga golfina & Lepidochelys olivacea & 1 & 25 \\
\hline & Pez vela & Istiophorus platypterus & 1 & 45 \\
\hline & & Subtotal & 176 & 4.549 \\
\hline & & Total & 1.304 & 43.719 \\
\hline
\end{tabular}




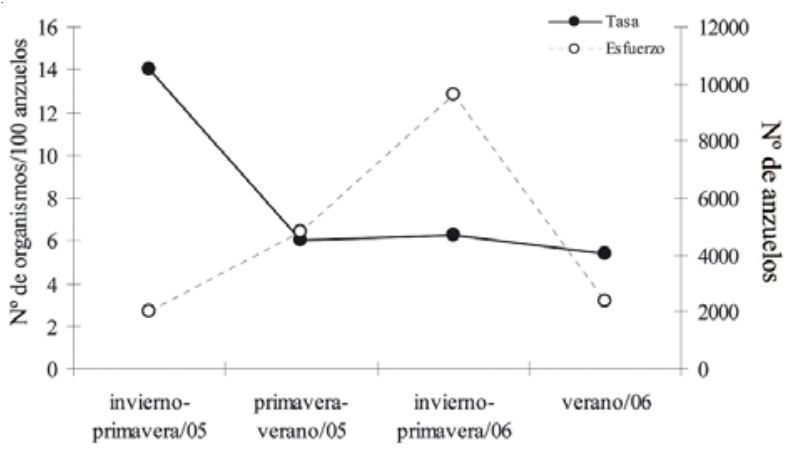

Figura 4

Tasa de captura total ( ${ }^{\circ}$ de organismos $/ 100$ anzuelos) y esfuerzo $\left(n^{\circ}\right.$ de anzuelos) aplicado por temporada de pesca

Catch rate of total catch ( $\mathrm{n}^{\circ}$ of organisms /100 hooks) and effort ( $n^{\circ}$ of hooks) applied by season fishing

\section{Comparación entre el anzuelo circular y el anzuelo recto}

El factor anzuelo no presentó diferencia significativa $(P>0,05)$ en la tasa de captura y peso de las seis especies consideradas en el análisis de varianza y de la captura total (Tabla 2); sin embargo los resultados mostraron que el anzuelo circular presentó valores promedios superiores a los valores promedios del anzuelo recto en la tasa de captura y peso del tiburón azul (7,5\% y 4,6\% respectivamente), dorado (6,3\% y 31\%), marlín rayado (6,9\% y 18,9\%), marlín azul (37,6\% y 67,4\%), captura total $(2,6 \%$ y $1,4 \%)$ y en la tasa de captura de tiburón mako (en 24,3\%). Mientras que el anzuelo recto tuvo valores promedios superiores a los valores promedios del anzuelo circular en la tasa de captura y peso del tiburón jaquetón (en 26,4\% y 20,7\% respectivamente) y en el peso del tiburón mako (en 1,2\%) pero sin diferencias significativas $(P>0,05)$ para todos los casos.

\section{Comparación entre la carnada de calamar y la de lisa}

Los resultados de la Tabla 2 muestran que la carnada presentó diferencia altamente significativa $(P<0,01)$ para la tasa de captura del dorado, la captura total y para el peso de la captura total, así como diferencia significativa $(P<0,05)$ para la tasa de captura del tiburón azul y tiburón jaquetón. De las comparaciones múltiples de la prueba de Tukey para la tasa de captura (Fig. 5), la carnada de calamar resultó con una media superior que la carnada de lisa para el tiburón azul (en 13,5\%), tiburón jaquetón (en 52,7\%), dorado (en 152\%) y el total de los organismos capturados (en 10,5\%).

Tabla 2

Resumen de los valores probabilidad estadística del modelo lineal general para determinar el efecto en la tasa de captura y peso debido a los diferentes anzuelos (circular y recto) y tipo de carnada (calamar y lisa)

Summary of statistical probability values from GLM to determine effects on catch rate and weight due to hooks type (circle hook and J-style hook) and bait type (jumbo squid and striped mullet)

\begin{tabular}{|c|c|c|c|c|c|c|c|c|c|c|c|c|c|c|c|}
\hline \multirow{2}{*}{ Factor } & \multirow{2}{*}{ g.l. } & \multicolumn{2}{|c|}{ Captura total } & \multicolumn{2}{|c|}{ Tiburón azul } & \multicolumn{2}{|c|}{ Tiburón jaquetón } & \multicolumn{2}{|c|}{ Tiburón mako } & \multicolumn{2}{|c|}{ Dorado } & \multicolumn{2}{|c|}{ Marlin rayado } & \multicolumn{2}{|c|}{ Marlin azul } \\
\hline & & Tasa & Peso & Tasa & Peso & Tasa & Peso & Tasa & Peso & Tasa & Peso & Tasa & Peso & Tasa & Peso \\
\hline VIA & 3 & $<, 0001$ & $<, 0001$ & $<, 0001$ & 0,001 & 0,000 & 0,037 & 0,465 & 0,455 & 0,000 & 0,038 & $<, 0001$ & $<, 0001$ & $<, 0001$ & $<, 0001$ \\
\hline VIA*LAN & 20 & 0,005 & 0,001 & 0,000 & 0,010 & 0,025 & 0,072 & 0,232 & 0,312 & 0,053 & 0,329 & 0,019 & 0,071 & 0,477 & 0,446 \\
\hline ANZ & 1 & 0,417 & 0,851 & 0,133 & 0,440 & 0,160 & 0,377 & 0,525 & 0,971 & 0,789 & 0,499 & 0,832 & 0,583 & 0,462 & 0,206 \\
\hline $\mathrm{VIA}^{*} \mathrm{ANZ}$ & 3 & 0,633 & 0,899 & 0,987 & 0,576 & 0,239 & 0,164 & 0,660 & 0,664 & 0,236 & 0,483 & 0,343 & 0,810 & 0,837 & 0,543 \\
\hline VIA*LAN*ANZ & 20 & 0,572 & 0,307 & 0,364 & 0,485 & 0,103 & 0,157 & 0,078 & 0,344 & 0,013 & 0,831 & 0,259 & 0,117 & 0,533 & 0,288 \\
\hline CAR & 1 & 0,005 & 0,002 & 0,013 & 0,561 & 0,017 & 0,068 & 0,220 & 0,353 & 0,001 & 0,094 & 0,380 & 0,220 & 0,694 & 0,737 \\
\hline VIA*CAR & 3 & 0,242 & 0,000 & 0,343 & 0,824 & 0,106 & 0,079 & 0,810 & 0,894 & 0,175 & 0,408 & 0,148 & 0,087 & 0,774 & 0,601 \\
\hline $\mathrm{LAN}^{*} \mathrm{CAR}$ & 23 & 0,245 & 0,871 & 0,404 & 0,350 & 0,276 & 0,966 & 0,769 & 0,449 & 0,103 & 0,999 & 0,019 & 0,015 & 0,989 & 0,997 \\
\hline VIA*LAN*CAR & 20 & 0,722 & 0,729 & 0,884 & 0,998 & 0,126 & 0,194 & 0,744 & 0,822 & 0,794 & 0,996 & 0,116 & 0,330 & 0,890 & 0,527 \\
\hline $\mathrm{ANZ}{ }^{*} \mathrm{CAR}$ & 1 & 0,415 & 0,660 & 0,136 & 0,540 & 0,129 & 0,111 & 0,979 & 0,220 & 0,984 & 0,977 & 0,600 & 0,910 & 0,694 & 0,670 \\
\hline VIA ${ }^{*} \mathrm{ANZ}{ }^{*} \mathrm{CAR}$ & 3 & 0,471 & 0,797 & 0,910 & 0,845 & 0,801 & 0,821 & 0,785 & 0,905 & 0,511 & 0,660 & 0,671 & 0,470 & 0,990 & 0,995 \\
\hline
\end{tabular}

VIA viaje (fishing trip); LAN lance (set); ANZ anzuelo (hook); CAR carnada (bit) 

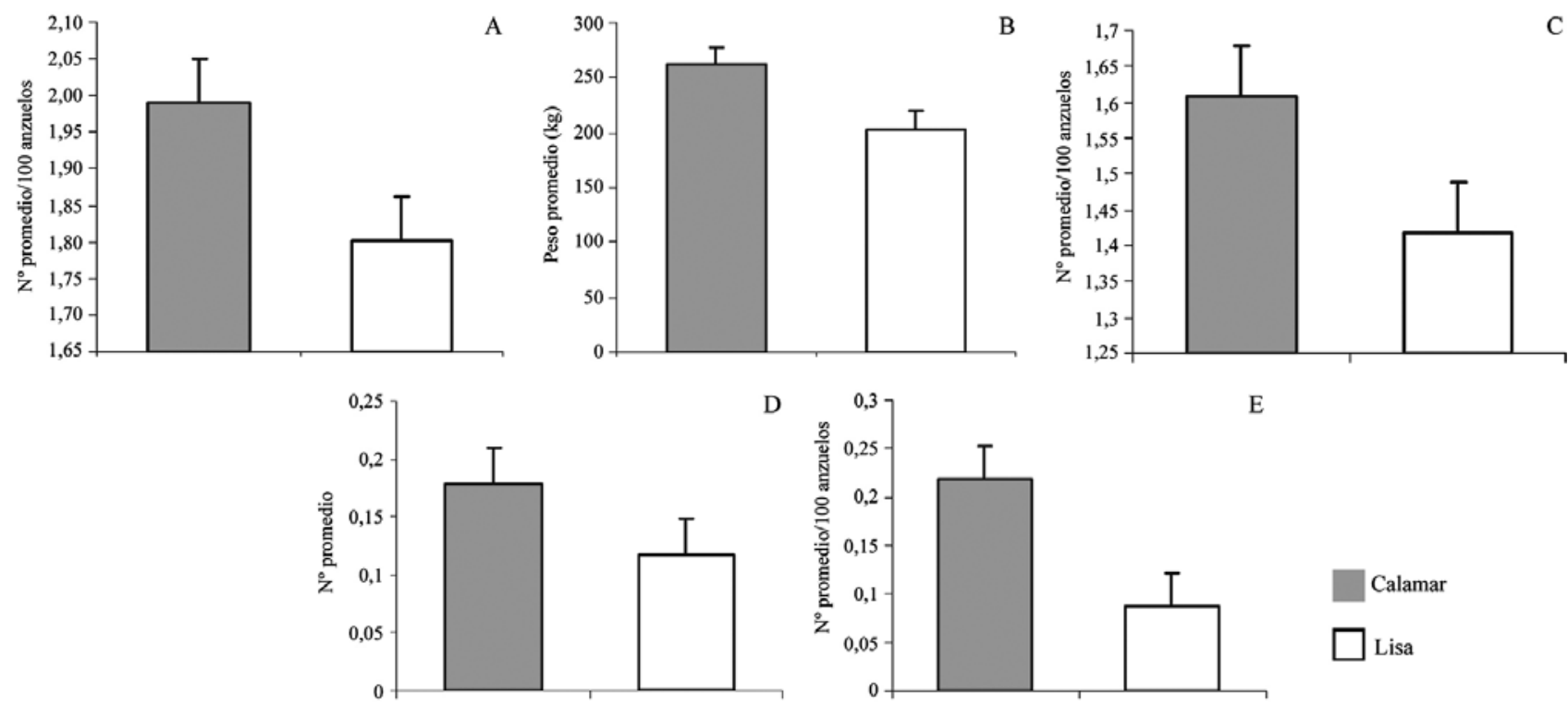

Figura 5

Valores medios ( \pm error estándar) entre dos tipo de carnada (calamar y lisa) en: A) tasa de captura total, B) peso de la captura total, $C$ ) tasa de captura de tiburón azul, D) tasa de captura de tiburón jaquetón, $E$ ) tasa de captura de dorado. $P=$ probabilidad estadística

Mean values ( \pm standard error) between two types of bit (squid and striped mullet) in: A) total catch rate, B) total catch weight, C) catch rate of blue shark, D) catch rate of silky shark, E) catch rate of dolphin fish. $P=$ probability

\section{Interacción entre anzuelo y la carnada}

Los resultados mostraron que no se presentó interacción del anzuelo y la carnada en la tasa de captura y peso de los datos de las seis especies y de la captura total considerados en el análisis (Tabla 2). Sin embargo, es conveniente destacar que aunque no se presentó interacción, la combinación anzuelo circular-calamar resultó con mayor valor promedio en la tasa de captura y peso de la captura total del tiburón azul y dorado; mientras que la combinación anzuelo recto-calamar presentó un valor promedio superior en la tasa de captura y peso del tiburón jaquetón y peso del tiburón mako. La combinación anzuelo recto-lisa fue superior en la tasa de captura del tiburón mako y marlín azul, así como en el peso del marlín rayado y marlín azul.

\section{Discusión}

Los barcos camaroneros adaptados con tambores hidráulicos para la operación del palangre pelágico en los que se realizó el experimento en condiciones de pesca comercial, tienen una autonomía de hasta 30 días. El tiempo de pesca en promedio es de 20 a 25 días con 15 a 16 lances aproximadamente que pueden incrementarse hasta 30 días con 24 lances. Debido a las condiciones de la pesca y a la resistencia natural de los pescadores de seguir un plan preestablecido de combinar los anzuelos con las carnadas de acuerdo con el diseño experimental implementado, el número de lances correctos por viaje no fue similar. Estas dificultades fueron la causa de la variación del esfuerzo (número de anzuelos) reportados en los resultados del experimento.

Del total de las especies capturadas de acuerdo con la definición de Hall (1996) los tiburones, las especies de peces picudos (marlín azul, marlín rayado, pez espada y pez vela) y el dorado, son la porción retenida que tiene valor comercial, en donde los tiburones son la especie objeto de captura primaria, mientras que las otras especies se consideran como especies objeto de captura secundaria. La presencia dominante del tiburón azul en la captura total $(73,6 \%)$, lo hace la especie más importante de las pesquerías de la costa occidental y sur de la península de Baja California, México (Carrera-Fernández 2004). Cabe destacar que cuando los volúmenes de captura de dorado, marlín azul, pez espada y pez vela son muy bajos, son retenidos por la tripulación para su consumo. El descarte ('by-catch') solo se presentó cuando los organismos capturados fueron dañados por ataque de tiburón o 
calamar gigante y por tales circunstancias no se pueden comercializar o no son aptos para el consumo humano, lo cual se presentó de manera esporádica durante todo el estudio. El wahoo (Acanthocybium solandrii), atunes (Thunnus spp.) y mantas (Mobula spp.) son retenidos en la embarcación para consumo de la tripulación por su bajo volumen de captura. Las especies de tortugas y rayas (Dasyatis violacea) representaron la porción de la captura total que fue liberada viva al mar. En el caso de las tortugas, se liberaron debido a disposiciones legales y las rayas por no presentar valor comercial.

La preocupación principal en las pesquerías comerciales es el problema del descarte ya que se considera como un desperdicio. Alverson et al. (1994) ha estimado un descarte promedio en un intervalo de 17,9 a 39,5 Tm con un promedio de 27 millones de Tm cada año en las pesquerías comerciales, siendo la mayor cantidad en el noroeste del Océano Pacífico. De los cuatro grupos de artes de pesca, las redes de arrastre para camarón son las que generan el mayor descarte, siguiendo el arrastre de fondo, palangres y trampas. De acuerdo con los resultados obtenidos en este estudio, se puede considerar que la pesquería de tiburón con el uso del palangre pelágico no presenta problemas de descarte muerto por el reducido porcentaje que se presentó y que prácticamente el total de descarte es de organismos vivos que se regresan al mar y no sobrepasan el 0,61\% de la captura total. Sin embargo, uno de los problemas principales de esta pesquería en México, es un conflicto de intereses entre la pesca comercial y la pesca deportiva al compartir el aprovechamiento del recurso de pelágicos mayores en los que se encuentran el marlín azul, marlín rayado, pez vela y dorados (Santana-Hernández 2001). Dentro de algunas medidas consideradas para regular estas pesquerías se encuentra el uso obligatorio del anzuelo circular con un tamaño mínimo de $64 \mathrm{~mm}$ de largo y $22 \mathrm{~mm}$ de abertura por lo menos en las profundidades más someras de operación, que corresponden al reinal más cercano a cada orinque del palangre en las pesquerías de mediana altura y de altura (Diario Oficial de la Federación 2007). Sin embargo, los resultados del estudio mostraron que no se presentaron diferencias significativas entre el anzuelo circular y el anzuelo recto en la tasa y peso de la captura de tiburones y especies asociadas. Para el caso de la captura de tiburón azul, estos resultados son consistentes con otros estudio como el de Yokota et al. (2006) en el Pacífico Norte frente a las costas de Japón, los cuales utilizaron un anzuelo atunero convencional (tamaño estándar japonés; 3,8 sun) y dos anzuelos circulares (4,3 sun y 5,2 sun); sin embargo, no encontraron diferencia significativa en la tasa de captura de tiburón azul. Esta especie de tiburón también fue dominante en sus capturas. Sin embargo, la tasa de captura fue afectada significativamente por las diferencias en la operación de pesca (número de lances).

El tipo de carnada que se utilizó en el presente estudio (calamar y lisa), registró diferencias significativas en los resultados de la captura, debido probablemente a la diferencia en las características de atracción entre el calamar y la lisa. El éxito de captura de las artes de pesca que utilizan carnadas, está basado en la actividad de los animales de búsqueda y captura de alimento (Løkkeborg 1994). El olor que desprende la carnada es una de sus principales características de atracción que permite estimular el comportamiento del pez en busca del alimento y provocar el contacto del animal con el arte de pesca para aumentar la probabilidad de su captura. Tradicionalmente se utiliza carnada natural en los palangres y la selección de su tipo está basada principalmente en la experiencia del pescador, su disponibilidad y su precio (Blaha 2003). En la pesca dirigida a tiburones en México es común utilizar la carnada de calamar, barrilete, lisa y macarela (SantanaHernández 1998). La media de la tasa de la captura total fue significativamente superior para la carnada de calamar y el experimento confirmó, lo que de manera empírica reportan los pescadores, que el calamar es más 'efectivo' para obtener mejores capturas. Sin embargo, desde el punto de vista del costo de la carnada, en comparación de precio por tonelada de ambas carnadas, el calamar cuesta aproximadamente $20 \%$ más que la lisa y por rendimiento en número de carnadas, la lisa rinde aproximadamente 2,25 veces más carnadas que el calamar por tonelada. Esto hace que la lisa, en comparación con el calamar represente menor costo y quizá esto sea una de las razones por lo que los pescadores, de manera rutinaria, en la pesca con el palangre pelágico encarnan los anzuelos con calamar como carnada principal e intercalan carnadas de lisa cada 3 ó 4 anzuelos.

Asimismo se debe considerar que la carnada juega un papel importante en el éxito de la pesca y depende también con los hábitos alimentarios de las especies a capturar. En este aspecto el calamar es una especie endémica relativamente abundante en la zona donde se realizó el estudio con mayores concentraciones en el Golfo de California, México (Nevárez-Martinez et al. 2000, Morales-Bojórquez 2001), teniendo importancia como disponibilidad de alimento (Olson \& Young 2007), lo que no sucede con la lisa que presenta una distribución en aguas costeras, principalmente lagunar-estuarinas (Ibáñez \& Lleonart 1996).

Los dorados son peces depredadores visuales que se alimentan de teleósteos y cefalópodos (Oxenford \& Hunte 
1999). Aguilar-Palomino et al. (1998) encontraron 51 especies presa en la dieta de dorados capturados frente a Cabo San Lucas, de las cuales el calamar gigante ( $D$. gigas) fue la presa principal. Quizás la disponibilidad abundante de calamar en la zona propicie una preferencia del dorado por esta presa, por lo que en las comparaciones de medias, la carnada de lisa resultó inferior en 60,4\% en la tasa de captura de dorado en comparación con el calamar. De acuerdo con los resultados de este estudio, se podría considerar que el uso de carnada de lisa en la pesquería de palangre pelágico propiciaría una reducción de la captura incidental de dorado, pero con el inconveniente de reducir la tasa de captura de tiburones que son la especie objeto de captura.

\section{Agradecimientos}

A los señores Jaime Andrés Osuna Magaña y J. Jesús Becerra Gutiérrez de la CANAIPESCA por las facilidades brindadas para realizar el estudio a bordo de sus embarcaciones palangreras. Agradecemos también al Ingeniero Pesquero Leobrando Pérez Jiménez por su participación a bordo de las embarcaciones en la realización del experimento. FGM agradece el apoyo del Instituto Politécnico Nacional (COFAA, EDI), así como a los evaluadores anónimos.

\section{Literatura citada}

Aguilar-Palomino B, F Galván-Magaña, LA AbitiaCárdenas, AF Muhlia-Melo, J Rodríguez-Romero. 1998. Aspecto alimentario del dorado Coryphaena hippurus Linnaeus, 1758 en Cabo San Lucas, Baja California Sur, México. Ciencias Marinas 24(3): 253-265.

Alverson DL, MH Freeberg, JG Pope \& SAMurawski. 1994. A global assessment of fisheries bycatch and discards. FAO Fisheries Technical Paper 339: 1-233.

Bigelow KA, CH Boggs \& X He. 1999. Environmental effects on swordfish and blue shark catch rate in the US North Pacific longline fishery. Fisheries Oceanography 8(3): 178-198.

Blaha FI. 2003. Bait for longlining fishery on snapper (Pagrus auratus). Alaska Sea Grant College Program. AK-SG-0301: 231-240.

Boggs HC. 1992. Depth, captures time, and hooked longevity of longline-caught pelagic fish: Time bait of fish with chips. Fishery Bulletin 90: 642-658.

Broadhurst MK \& HV Hazin. 2001. Influences of type and orientation of bait on catch of swordfish (Xiphias gladius) and other species in artisanal sub-surface longline fishery off northeastern Brazil. Fisheries Research 53: 169-179.

Carrera-Fernández FM. 2004. Biología reproductiva del tiburón azul Prionace glauca (Linnaeus, 1758) en la costa occidental de Baja California Sur, México. Tesis de Maestría en Ciencias, CICIMAR-IPN, México, 67 pp.
CONAPESCA-INP. 2004. Plan de acción nacional para el manejo y conservación de tiburones, rayas y especies afines en México, 80 pp. Comisión Nacional de Acuacultura y Pesca e Instituto Nacional de la Pesca, Secretaría de Agricultura, Ganadería, Desarrollo Rural, Pesca y Alimentación. Mazatlán.

Diario Oficial de la Federación. 2007. Norma oficial mexicana. NOM-029-PESC-2006. Pesca responsable de tiburones y rayas. Especificación para su aprovechamiento, 43 pp. Secretaría de Gobernación, Mexico.

Fréon P \& OL Misund. 1999. Dynamics of pelagic fish distribution and behaviour: Effect of fisheries and stock assessment, 348 pp. Fishing New Books, London.

Hall MA. 1996. On bycatches. Review of Fish Biology and Fisheries 6: 319-352.

Herrera HJG \& SA Barreras. 2001. Análisis estadísticos de experimentos pecuarios (utilizando el programa SAS), Manual de procedimientos, 100 pp. Colegio de Posgraduados, México.

Ibáñez AAL \& J LLeonart. 1996. Relative growth and comparative morphometrics of Mugil cephalus L. and $M$. curema V. in the Gulf of Mexico. Scientia Marina 60(2-3): 361-368.

Jacobsen JH \& J Joensen. 2004. Comparison of bait in longline fishery. BSc. Thesis, University of Faroe Island, Denmark, 54 pp.

Johnstone ADF \& AD Hawkins. 1981. A methods testing the effectiveness of different fishing baits in the sea. Scottish Fisheries Information Pamphlet Number 3: 1-7.

Kaimmer S. 1999. Direct observation on the hooking behavior on Pacific halibut, Hippoglossus stenolepis. Fishery Bulletin 97: 873-883.

Kerstetter DW \& JE Graves. 2006. Effects of the circle versus J-style hooks on target and no-target species in a pelagic longline fisheries. Fisheries Research 80: 239-250.

Løkkeborg S. 1994. Fish behavior and longline. En: Fernö A \& S Olsen (eds). Marine fish behavior in capture and abundance estimation, pp. 9-27. Fishing New Books. Oxford.

Løkkeborg S \& Å Bjordal. 1992. Species and size selectivity in long line fishing: a review. Fisheries Research 13: 311-322.

Løkkeborg S \& T Pina. 1997. Effects of setting time, setting direction and soak time on longline catch rates. Fisheries Research 32: 213-222.

Montgomery DC. 1976. Design and analysis of experiments, 418 pp. John Wiley \& Sons, New York.

Morales-Bojórquez E, MA Cisneros-Mata, MO NevárezMartínez \& AHernández-Herrera. 2001. Review of stock assessment and fishery biology of Dosidicus gigas in the Gulf of California, Mexico. Fisheries Research 54: 83-94.

Nevárez-Martínez MO, A Hernández-Herrera, E MoralesBojórquez, A Balmori-Ramírez, MA Cisneros-Mata \& R Morales-Azpeitia. 2000. Biomass and distribution of the jumbo squid (Dosidicus gigas; d'Orbigny, 1835) in the 
Gulf of California, Mexico. Fisheries Research 49: 129140.

Olson RJ \& JW Young (eds). 2007. The role of squid in open ocean ecosystems. Report of a GLOBEC-CLIOTOP/PFRP workshop, 16-17 November 2006, Honolulu, Hawaii, USA. GLOBEC Report 24: 1-94.

Oxenford HA \& W Hunte. 1999. Feeding habitat of the dolphinfish (Coryphaena hippurus) in the eastern Caribbean. Scientia Marina 63(3-4): 303-305.

Santana-Hernández H. 2001. Estructura de la comunidad de pelágicos mayores capturados con palangre en el pacífico mexicano (1983-1996) y su relación con la temperatura superficial del mar. Tesis Doctoral, Posgrado Interinstitucional de Ciencias Pecuarias, Facultad de Medicina Veterinaria y Zootecnia, Universidad de Colima, México, 122 pp.

Santana-Hernández H, R Macías-Zamora \& JJ ValdezFlores. 1998. Selectividad del sistema de palangre utilizado por la flota mexicana en la Zona Económica Exclusiva. Ciencias Marinas 24(2): 193-210.

Yokota K, M Kiyota \& H Minami. 2006. Shark catch in a pelagic longline fishery: comparison of circle and tuna hooks. Fisheries Research 81: 337-341.

Recibido el 13 de febrero de 2008 y aceptado el 20 de enero de 2009 